\section{Magnetic field in the primitive solar nebula}

METEORITES which fall to Earth's surface generally carry permanent magnetisation which probably was acquired early in their history ${ }^{1}$. In particular, carbonaceous chondrites, which are generally assumed to be virtually unaltered relicts from the earliest Solar System processes of condensation and accretion ${ }^{2}$, have apparently been magnetised in magnetic fields with intensities in the range $0.1-10 \mathrm{G}$ (refs $1,3,4$ ). The origin of the magnetising field has remained obscure; explanations proposed to account for the field, include a strong central solar field in the early Sun, an intense solar wind magnetic field, magnetic fields generated in large parent objects-ancestral to the meteoritesand the interstellar magnetic field transiently compressed during the Solar System's formation. So far as we know, none of these mechanisms has been shown quantitatively capable of accounting for measured meteoritic remanence ${ }^{5}$. We suggest here that the magnetic field recorded in the remanence of carbonaceous chondrites may have been produced by a self-excited hydromagnetic dynamo in the gaseous preplanetary nebula from which the Solar System is thought to have formed.

The Solar System is generally thought to have formed from the collapse of gaseous interstellar matter which, because of the system's angular momentum, settled into a disk shaped nebula. Several models to describe the evolution of such a nebula have been computed ${ }^{6-10}$. While these models are not unique, they do give some indication of the processes and conditions involved in the early evolution of the Solar System. For specificity, we will use the models recently computed by Cameron ${ }^{10}$, which consist of turbulent and differentially rotating, gaseous disks having characteristic radial scales of several astronomical units and characteristics half thicknesses of a few times $10^{12} \mathrm{~cm}$. In the models the gas density characterising the region at several astronomical units from the centre is $\sim 3 \times 10^{-10} \mathrm{~g} \mathrm{~cm}^{-3}$ and the characteristic turbulent fluid velocity is $\sim 4 \times 10^{4} \mathrm{~cm} \mathrm{~s}^{-1}$.

Turbulent motions in such a differentially rotating gas nebula can generate a large-scale magnetic field through hydromagnetic dynamo action if the electrical conductivity of the gas is sufficiently high. At the low temperatures and high gas densities typical of a preplanetary nebula, the electron density in thermodynamic equilibrium is too low to support hydromagnetic processes. These processes then require nonequilibrium effects to raise the electron density and electrical conductivity. The recent discovery by Lee et al. ${ }^{11}$ of anomalously large concentrations of ${ }^{26} \mathrm{Mg}$ in some meteorite samples suggests that the short-lived radioactive isotope ${ }^{26} \mathrm{Al}$ may have been present in abundant quantities in the nebular material-the ${ }^{26} \mathrm{Al}$ produced in a nearby supernova thought to have precipitated the Solar System's formation. Cameron and Truran ${ }^{12}$ have estimated that $\sim 2 \%$ of the Solar System's heavy elements may have been inserted into the nebula by such an event. Ionising radiation from the decay of short-lived isotopes drives up the electrical conductivity of the nebular gas. In conditions which characterise nebular models the conductivity may be so large that the magnetic Reynolds number exceeds unity, thus coupling the behaviour of magnetic fields to the fluid motion.

In a turbulent, differentially rotating gas the efficacy of the fluid motions for generating a magnetic field can be expressed through a dimensionless dynamo number ${ }^{13,14}$

$$
N \equiv \gamma \Gamma \delta^{3} / \eta^{2}
$$

where $\gamma$ measures the shear produced by differential rotation, $\Gamma$ measures the helicity of the turbulent convection, $\delta$ is the physical scale of the magnetic field, and $\eta$ is the magnetic diffusivity $\left(\eta=c^{2} / 4 \pi \sigma ; c\right.$ is the speed of light and $\sigma$ is the electrical conductivity).

Parker has investigated the regenerative modes of a hydromagnetic dynamo operating in a differentially rotating turbulent disk of $\operatorname{gas}^{16}$ and has found that magnetic fields are produced when $N \geqslant 6$. It will be shown elsewhere ${ }^{5}$ that this condition is satisfied in the model nebula when $\sigma \geqslant 400 \mathrm{~s}^{-1}$. Consolmagno and Jokipiii ${ }^{15}$ suggest that ionisation produced by the decay of ${ }^{26} \mathrm{Al}$ can produce an electrical conductivity of the order of $10^{3} \mathrm{~s}^{-1}$ in the nebula, thus satisfying the condition for magnetic field generation.

The maximum field intensity that might be realised by the dynamo production process can be estimated. We will consider two dynamical mechanisms that limit the strength of the field. First, because the Coriolis force provides the ordering influence that produces efficient magnetic field generation in the dynamo process, an estimate of the maximum realisable field intensity can be obtained by setting the Coriolis force equal to the Lorentz force produced by the magnetic stress ${ }^{14}$. Thus, in order of magnitude

$$
\rho V \Omega \sim\left\langle B^{2}\right\rangle / 4 \pi \delta
$$

Taking, in equation (2), the density $\rho \simeq 3 \times 10^{-10} \mathrm{~g} \mathrm{~cm}^{-3}$, the characteristic turbulent velocity $V \simeq 4 \times 10^{4} \mathrm{~cm} \mathrm{~s}^{-1}, \quad \delta \simeq$ $5 \times 10^{12} \mathrm{~cm}$, the disk scale height, and $\Omega$ the Keplerian angular velocity at several astronomical units from the Sun, we find for the characteristic saturation field intensity

$$
\left\langle B^{2}\right\rangle^{1 / 2} \sim 5 \mathrm{G}
$$

This is the same order of magnitude as the magnetic field which would be necessary to account for meteorite magnetic remanence.

Another mechanism which limits the field strength is ambipolar diffusion, through which the ionised gas and magnetic field slips past the neutral material ${ }^{17}$. Using the results of Consolmagno and Jokipii ${ }^{15}$ and equating the ambipolar diffusion time to the turnover time of the turbulent fluid motions one finds that ambipolar diffusion limits the further growth of the magnetic field when the field intensity rises to the order of $1 \mathrm{G}$.

The values derived here are to a large extent model-dependent and will evolve along with our ideas about the characteristics of a preplanetary nebula. However, these crude and preliminary calculations, taken with the measured remanence of primitive meteorites, suggest that a large-scale magnetic field was generated by gas motions in the preplanetary solar nebula. The intensity of this field may have been as high as $1-10 \mathrm{G}$.

Such an intense field could have had important dynamical influences on the evolution of the gaseous nebula. It is easy to see that the timescale for redistribution of angular momentum (and thus mass) driven by the magnetic stress, arising from field intensities in the range $1-10 \mathrm{G}$, is in the range $10^{2}-10^{4} \mathrm{yr}$. This is the same range estimated for the evolutionary time scale of the primitive solar nebula ${ }^{10}$.

I thank J. R. Jokipii for discussions. This research was supported in part by NASA.

\section{Department of Planetary Sciences}

E. H. LEVY

and Lunar and Planetary Laboratory,

\section{University of Arizona,}

Tucson, Arizona 85721

Received 18 July; accepted 9 October 1978

1. Brecher, A. in Comets, Asteroids, Meteorites (ed. Delsemme, A. H.) 415 (University of Toledo Press, 1977).

2. Wasson, J. T. Meteorites (Springer, Berlin, 1974).

3. Butler, R. F. Earth planet. Sci. Lett. 17, 120 (1972).

4. Lanoix, M., Strangway, D. W. \& Pearce, G. W. Lunar planet. Sci. IX, Abstr. (NASA Washington, D.C. 1978)

5. Levy, E. H. \& Sonett, C. P. in Protostars and Planets (ed. Gehrels, T.) (University of Arizona Press, in the press)

6. Safronov, V. S. Evolution of the Protoplanetary Cloud, (NASA translation, TTF 667, Washington, D.C. 1972).

7. Schatzman, E. Physics of the Solar System, 413 (NASA TMX 65702 1971)

8. Cameron, A. G. W. \& Pine. M. R. Icarus 18, 377 (1973).

9. Cameron, A. G. W. Proc. NATO Advanced Study Institute on the Origin of the Solar System (in the press).

10. Cameron, A. G. W. Center for Astrophysics Preprint No. 841 (1977).

11. Lee, T., Papanastassiou, D. A. \& Wasserburg, G. J. Geophys. Res. Lett. 3, 109 (1976).

12. Cameron, A. G. W. \& Truran, J. W. Icarus 30, 447 (1977)

13. Parker, E. N. Astrophys. J. 162, 665 (1970).

14. Levy, E. H. A. Rev. Earth planet. Sci. 4, 159 (1976).

15. Consolmagno, G. J. \& Jokipii, J. R. Moon and Planets (in the press).

16. Parker, E. N. Astrophys. J. 163, 255 (1971)

17. Spitzer, L., Jr Diffuse Matter in Space (Wiley New York, 1968). 